\title{
Parity-Violating Interactions and Currents in the Deuteron
}

\author{
R. Schiavilla \\ Jefferson Laboratory \\ Newport News, VA 23606 \\ and \\ Physics Department \\ Old Dominion University \\ Norfolk, VA 23529 \\ J. Carlson and M. Paris \\ Theoretical Division \\ Los Alamos National Laboratory \\ Los Alamos, NM 87545
}

\begin{abstract}
We investigate parity-violating asymmetries in $\vec{n} p$ radiative capture at thermal neutron energies and in deuteron electro-disintegration in quasi-elastic kinematics, using the DDH model for the parity-violating nucleon-nucleon interaction. We find dramatic cancellations between the asymmetries induced by the parity-violating interaction and those arising from the associated parity-violating pion-exchange currents. In the $\vec{n} p$ capture, the model-dependence of the result is nevertheless quite small, because of constraints arising through the Siegert evaluation of the relevant $E_{1}$ matrix elements. In quasi-elastic electron scattering these processes are found to be insignificant compared to the asymmetry produced by $\gamma-Z$ interference on individual nucleons. These two experiments, then, provide clean probes of different aspects of weak-interaction physics associated with parity violation in the $n p$ system.
\end{abstract}




\section{INTRODUCTION}

Two-nucleon experiments are the clearest probes of hadronic parity violation. A recent experiment has measured the longitudinal asymmetry in $\overrightarrow{p p}$ elastic scattering [1], while another one is underway to measure the photon asymmetry in low-energy $\vec{n} p$ radiative capture [2]. This experiment has been designed to provide a definitive measurement of the weak $\pi N N$ coupling constant, which determines the longest-range part of the parityviolating nucleon-nucleon interaction.

We investigate this process using the Desplanques, Donoghue, and Holstein (DDH) meson-exchange model of the parity-violating $N N$ interaction [3]. We separately evaluate the contributions from the hadronic weak interaction and the associated two-body currents. These currents play an important role, reducing dramatically the measured asymmetry. We

also consider the model-dependence of the full result by using different modern models of the strong interaction. Even though there are significant cancellations between the two terms above, the final model dependence is quite small, and in fact similar in size to the estimated contribution of short-range mechanisms.

It has been speculated that these interactions and currents could potentially also play a role in the parity-violating quasi-elastic electro-disintegration of the deuteron, recently measured in the SAMPLE experiments at the MIT-Bates facility [4, 5]. Indeed, their contributions could cloud the interpretation of the experimental asymmetry in terms of singlenucleon properties. However, we find that these two-nucleon mechanisms lead, for any reasonable value of the $\pi N N$ coupling constant, to very small asymmetries when compared to those originating from $\gamma-Z$ interference. Therefore, these electron scattering results can be reliably used to extract single-nucleon properties.

A more complete account of the calculations carried out so far will be published elsewhere [6]. In the present letter, we only report the main results.

\section{ASYMMETRY IN $\vec{n} p$ RADIATIVE CAPTURE}

The parity-violating (PV) observable in the $\vec{n} p$ radiative capture-the asymmetry in the angular distribution of the outgoing photon with respect to the direction of the initial neutron polarization-is very sensitive to the weak parity-violating $\pi N N$ coupling [7, 8]. Since this 
coupling contributes to the longest-range part of the interaction, it provides a window into the hadronic weak interaction in a manner similar to the role the standard $\pi N N$ coupling provides in strong-interaction physics. Its experimental determination, however, has proven to be very difficult. Measurements of the circular polarization of gamma-ray decays in ${ }^{18} \mathrm{~F}$ [9] have been interpreted to indicate a very small $\pi N N$ coupling as compared to theoretical expectations based upon hadronic models [3], while measurements in atomic ${ }^{133} \mathrm{Cs}$ seem to favor a much larger value [10]. The analysis of both these experiments is complicated by the difficulty of handling reliably, from a theoretical stand-point, the many-body aspects of the problem in these complex systems. These issues have been addressed in Refs. [11 and [12]. More recently, PV asymmetry measurements of neutron resonances in compound nuclei seem to require even larger values of the weak $\pi N N$ coupling constant [13].

For these reasons, an experiment measuring the photon asymmetry in $\vec{n} p$ capture has been undertaken at the LANSCE facility [2]. As mentioned above, previous studies of this process [7, 8] have shown that this asymmetry is very sensitive to the weak $\pi N N$ coupling constant, while essentially unaffected by short-range contributions. Here we investigate the model dependence of this result by considering several different high-quality interactions fit to the strong-interaction data. In addition, we consider the individual contributions to the final result, including processes where the photon couples at all points to the exchanged (virtual) pion.

We employ the standard DDH meson-exchange model of the (PV) NN interaction, solving the full Schrödinger equation for the scattering state as well as the deuteron bound state. The equations and relevant mixing parameters in the $S$-matrix have been discussed in considerable detail in Ref. [14 for the case of $\overrightarrow{p p}$ elastic scattering. While the short-range contributions to the PV interaction should not be viewed as resulting only from the exchange of single mesons, the seven parameters of the DDH model can still be employed to characterize all the low-energy PV mixings. For example, two-pion exchange could play a role [15], however we assume that its effects can be included, at least at low energy, through the present combination of pion- and short-range terms. The complete expressions for the $S$-matrix and contributions of the various terms will be presented in Ref. [6].

We calculate the asymmetry for the AV18 [16], Bonn-CD [17] and Nijmegen-I (NIJMI) [18] interactions. Each strong interaction model has associated exchange currents. For the AV18 we include currents from the momentum-independent terms ( $\pi$ - and $\rho$-currents) as 
well as terms from the momentum-dependent terms in the interaction. Further discussion of the AV18 currents is given below, for a review see Ref. [19]. For the Bonn-CD and NIJM-I interactions, we include $\pi$ - and $\rho$-currents with the cutoffs from the Bonn-CD model $\left(\Lambda_{\pi}=1.72 \mathrm{GeV}\right.$ and $\left.\Lambda_{\rho}=1.31 \mathrm{GeV}\right)$. Contributions from other meson exchanges in the Bonn and Nijmegen models have been neglected. In all calculations, the currents associated with $\Delta$ excitation and $\omega \pi \gamma$ transition have been included [19].

The total cross section at thermal neutron energies is due to the well-known $M_{1}$ transition connecting the parity-conserving $(\mathrm{PC}){ }^{1} \mathrm{~S}_{0} n p$ state to the $\mathrm{PC}$ deuteron state. The calculated values for each model are given in Table I, both for one-body (impulse) currents alone and for the one- and two-body currents. In each case the largest two-body contribution, approximately two-thirds of the total, comes from the currents associated with pion exchange. The total cross section is in good agreement with experimental results, which are variously quoted as 334.2(0.5) mb [20 or 332.6(0.7) mb 21]. It would be possible to adjust, for example, the transition magnetic moment $\mu_{\gamma N \Delta}$ of the $\Delta$-excitation current to precisely fit one of these values, here we simply choose a $\mu_{\gamma N \Delta}$ of 3 n.m., which is consistent with an analysis of $\gamma-N$ data at resonance.

The PV asymmetry arises from an interference between the $M_{1}$ term above and the $E_{1}$ transition, connecting the ${ }^{3} \mathrm{P}_{1} \mathrm{PV} n p$ state to the $\mathrm{PC}$ deuteron state and the ${ }^{3} \mathrm{~S}_{1} \mathrm{PC} n p$ state to the ${ }^{3} \mathrm{P}_{1} \mathrm{PV}$ deuteron state. The PV components of the wave functions are generated by the DDH potential, including $\pi$-, $\rho$-, and $\omega$-exchange mechanisms. In this work we have taken the linear combination of $\rho$ - and $\omega$-weak coupling constants corresponding to $p p$ scattering from an earlier analysis [14 of these experiments. The remaining couplings have been taken from the DDH "best guess" estimates. Finally, as in the earlier analysis of $\overrightarrow{p p}$ scattering, the cutoff values in the meson-exchange interaction are taken from the Bonn-CD potential $\left(\Lambda_{\pi}=1.72 \mathrm{GeV}, \Lambda_{\rho}=1.31 \mathrm{GeV}\right.$, and $\left.\Lambda_{\omega}=1.50 \mathrm{GeV}\right)$.

With these couplings, we obtain the asymmetries also listed in Table I. The results are consistent with earlier [7] and more recent [22] estimates, and all agree within a few per cent, which is also the magnitude of the contributions from the short-range terms. The $E_{1}$ transition has been calculated in the long-wavelength approximation (LWA) with the Siegert form of the $E_{1}$ operator, thus eliminating many of the model dependencies and leaving only simple (long-range) matrix elements. We have explicitly calculated corrections beyond the LWA $E_{1}$ terms, and found them to be quite small. 
We have also calculated the $E_{1}$ contributions with the same current operator used to calculate the $M_{1}$ matrix element. To the extent that retardation corrections beyond the LWA of the $E_{1}$ operator are negligible [23], this should produce identical results provided the current is exactly conserved. In order to satisfy current conservation, currents from both the strong (PC) and weak (PV) interactions are required. In the following we keep only the $\pi$-exchange term in the DDH interaction with their "best guess" for the weak $\pi$ - $N$ coupling constant, and use the AV18 strong-interaction model.

The PC $\pi$ - and $\rho$-currents are derived from the $v_{6}$ part of the AV18 interaction and by construction exactly satisfy current conservation with it [24]. The same prescription is used to generate conserved PV $\pi$-currents from the DDH interaction in the presence of a shortrange cutoff (as is the case here). However, the PC currents originating from the momentumdependent terms of the AV18 are strictly not conserved. For example, the currents from the $\mathbf{L}^{2}$ and $(\mathbf{L} \cdot \mathbf{S})^{2}$ components of the AV18 are constructed by minimal substitution [24]. While this procedure leads to conserved currents for the isospin-independent $\mathbf{L}^{2}$ and $(\mathbf{L} \cdot \mathbf{S})^{2}$ terms, it is not adequate for their isospin-dependent counter-parts, as one can easily surmise by considering their commutator with the charge density operator. This commutator requires the presence of terms with the isospin structure $\left(\tau_{i} \times \tau_{j}\right)_{z}$, which cannot be generated by minimal substitution (so-called internal radiation contributions [25]). This issue will be discussed more thoroughly in Ref. [6].

The currents associated with the momentum-dependent terms, however, while small, play here a crucial role, because of the large cancellation between the (PC) $\pi$ - and $\rho$-currents from the AV18 and the (PV) $\pi$-currents from the DDH. This point is illustrated in Table II. Note that the PC currents from $\Delta$-excitation and $\omega \pi \gamma$ transition mechanisms are transverse and therefore do not affect the $E_{1}$ matrix element. However, they slightly reduce the PV asymmetry since their contributions increase the $M_{1}$ matrix element by $\simeq 1 \%$. They are not listed in Table II.

The asymmetry is given by the sum of the two columns in Table II, namely $+0.17 \times 10^{-8}$ (last row). This value should be compared to $-5.02 \times 10^{-8}$, obtained with the Siegert form of the $E_{1}$ operator for the same interactions (and currents for the $M_{1}$ matrix element). As already mentioned, we have explicitly verified that retardation corrections in the $E_{1}$ operator are too small to account for the difference. Thus this difference is to be ascribed to the lack of current conservation, originating from the isospin- and momentum-dependent terms of 
the AV18.

To substantiate this claim, we have carried out a calculation based on a $v_{6}$ reduction [26] of the AV18, constrained to reproduce the binding energy of the deuteron and some of the $N N$ phase shifts. In this case, the resulting PC currents (only the $\pi$ - and $\rho$-exchange terms are present) are exactly conserved. The results are listed in Table III. The remaning $\simeq 2.7 \%$ difference between the Siegert result and the full calculation is presumably due to numerical inaccuracies as well as additional corrections from retardation corrections and higher order multipoles. Both of these effects are included in the full calculation.

\section{ASYMMETRY IN $d\left(\vec{e}, e^{\prime}\right) p n$ ELECTRO-DISINTEGRATION}

The SAMPLE experiment 4, 5] has measured the parity-violating asymmetry in polarized electron quasi-elastic scattering on the deuteron. This asymmetry has two distinct contributions: one associated with interference of the $\gamma$ - and $Z$-exchange amplitudes, and the other induced by PV NN interactions. The first contribution was recently studied in Ref. [27], where it was shown that two-body terms in the nuclear electromagnetic and weak neutral currents only produce $1-2 \%$ corrections to the asymmetry due to the corresponding single-nucleon currents.

In the present study, we investigate the asymmetry originating from hadronic weak interactions. We update and sharpen earlier predictions obtained in Refs. [28, 29]-these studies did not include the effects of PV currents.

The expression for the asymmetry from gamma-Z interference is given in Ref. [27], while that due to the hadronic weak interaction reads [6]

$$
A\left(q, \omega, \theta_{e}\right)=\frac{v_{T}^{\prime} R_{T}^{\prime}(q, \omega)}{v_{L} R_{L}(q, \omega)+v_{T} R_{T}(q, \omega)},
$$

where $v_{L}, v_{T}$, and $v_{T}^{\prime}$ denote electron kinematical factors, $R_{L}$ and $R_{T}$ are the standard longitudinal and transverse response functions (explicit expressions for these and the $v$ factors are given in Ref. [27]), and $R_{T}^{\prime}$ is the PV response function, defined as (again, in the notation of Ref. [27])

$$
R_{T}^{\prime}(q, \omega)=\overline{\sum_{i}} \sum_{f} \delta\left(\omega+m_{i}-E_{f}\right) \operatorname{Im}\left[\mathbf{j}_{f i}(\mathbf{q}) \times \mathbf{j}_{f i}^{*}(\mathbf{q})\right]_{z}
$$


Here the three-momentum transfer $\mathbf{q}$ has been taken to define the spin-quantization axis (the $z$-axis), and $\mathbf{j}_{f i}(\mathbf{q}) \equiv\langle f|\mathbf{j}(\mathbf{q})| i\rangle$ are the matrix elements of the electromagnetic current. In terms of electric and magnetic multipole operators, the cross product above is expressed as

$$
\operatorname{Im}\left[\mathbf{j}_{f i}(\mathbf{q}) \times \mathbf{j}_{f i}^{*}(\mathbf{q})\right]_{z} \propto \sum_{l \geq 1} \operatorname{Re}\left[\left\langle J_{f}\left\|M_{l}(q)\right\| J_{i}\right\rangle\left\langle J_{f}\left\|E_{l}(q)\right\| J_{i}\right\rangle^{*}\right],
$$

and therefore vanishes unless (i) the initial and/or final states do not have definite parity (as is the case here because of the presence of PV $N N$ interactions) and/or (ii) the electric and magnetic multipole operators have unnatural parities $(-)^{l+1}$ and $(-)^{l}$, respectively, because of PV electromagnetic currents, such as the one-body anapole current [30] and the two-body currents due to PV $N N$ interactions $[6]$.

The one-body anapole current is taken as [30]

$$
\mathbf{j}_{P V}^{(1)}(\mathbf{q})=\frac{Q^{2}}{2 m^{2}} \sum_{i}\left[a_{S}\left(Q^{2}\right)+a_{V}\left(Q^{2}\right) \tau_{i, z}\right]_{i} \mathrm{e}^{\mathrm{i} \mathbf{q} \cdot \mathbf{r}_{i}},
$$

where $Q^{2}$ is the squared four-momentum transfer $\left(Q^{2}=q^{2}-\omega^{2}\right)$, and the isoscalar and isovector anapole form factors are normalized as

$$
a_{S, V}(0)=\frac{h_{\pi} g_{\pi}}{4 \sqrt{2} \pi^{2}} \alpha_{S, V},
$$

with $\alpha_{S}=1.6$ and $\alpha_{V}=0.4$ from a calculation of pion-loop contributions [30]. Here $h_{\pi}$ and $g_{\pi}$ are weak and strong $\pi N N$ coupling constants, respectively. More recent estimates of the nucleon anapole form factors give somewhat smaller values for $\alpha_{S, V}$ [31, 32, 33]. A complete treatment would require estimates of short-distance contributions [34] and electroweak radiative corrections, we postpone that discussion to a later paper.[6]

Only the PV two-body current associated with the $\pi$-exchange term in the DDH interaction is included in the present calculations (in addition, of course, to the PC two-body currents discussed in the previous section). The PV currents from $\rho$ and $\omega$ exchanges have not yet been considered [6, 12], but are not expected to play a significant role. One should note that at the higher momentum transfers of interest here, 100-300 MeV/c, relevant for the SAMPLE experiments, it is not possible to include the currents through the Siegert theorem, they must be calculated explicitly. 
The calculation proceeds as discussed in Ref. [27]. We have used the AV18 or BonnCD models (and associated currents) in combination with the full DDH interaction (with coupling and cutoff values as given in the previous section). The final state, labeled by the relative momentump, pair spin and z-projection $S M_{S}$, and pair isospin $T\left(M_{T}=0\right)$, is expanded in partial waves; PC and PV interaction effects are retained in all partial waves with $J \leq 5$, while spherical Bessel functions are employed for $J>5$. In the quasi-elastic regime of interest here, it has been found that interaction effects are negligible for $J>5$.

In Fig. 1 we show the asymmetry for one of the SAMPLE kinematics for the AV18 plus full DDH interaction. The total asymmetry is given by the sum of the three contributions shown. The contribution labeled "DDH interaction" is that originating directly from the DDH interaction, while that labeled "PV $\gamma$-couplings" is from the (one-body) anapole term and the two-body pion current. Results at lower $Q^{2}$ are similar. The Bonn-CD model leads to predictions, for both the asymmetry and inclusive cross section, which are very close to those obtained with the AV18 [6].

In Fig. 2 we plot the asymmetry at the top of the quasi-elastic peak as a function of the four-momentum transfer. SAMPLE kinematics have $Q^{2}$ equal to $0.1(\mathrm{GeV} / \mathrm{c})^{2}$ and 0.043 $(\mathrm{GeV} / \mathrm{c})^{2}$, respectively. The leading $\gamma-Z$ term decreases in magnitude with $Q^{2}$, as expected. This term does not contribute, of course, for the real photons produced in the $\vec{n} p$ radiative capture. Also shown in the plot is the asymmetry obtained by retaining only the pion term in the DDH interaction, with a coupling given by the "best guess".

These results demonstrate that, in the kinematics of the SAMPLE experiments, the asymmetry from the $\gamma-Z$ coupling is two-orders of magnitude larger than that associated with the PV hadronic weak interaction. Hence even the largest estimates of the weak $\pi N N$ coupling constant will not affect extractions of nucleon matrix elements.

\section{CONCLUSION}

Parity-violating asymmetries in $n p$ radiative capture and deuteron electro-disintegration have been investigated within the framework of the DDH model of the parity-violating hadronic weak interaction. We find that the model-dependence of the $n p$-capture asymmetry is quite small, at a level similar to the expected contributions of the short-range parts of the interaction. This process is in fact dominated by the longer-range pieces of the interaction 
associated with pion-exchange, and hence this experiment is a clean probe of that physics.

Similarly, we find that the SAMPLE experiment measuring the asymmetry in electron scattering on the deuteron is a very clean probe of nucleon properties. The processes associated with two-nucleons, including parity violation in the deuteron and scattering wave functions, and the currents associated with these interactions, play a very small role at reasonable values of the momentum transfer. These two experiments, then, probe distinct aspects of weak-interaction physics.

We like to thank C.-P. Liu, G. Prézeau, and M.J. Ramsey-Musolf for making available to us the results of their calculation of the asymmetry in quasi-elastic deuteron electrodisintegration prior to publication. The work of J.C. and M.P. was supported by the U.S. Department of Energy under contract W-7405-ENG-36, while that of R.S. was supported by the U.S. Department of Energy contract DE-AC05-84ER40150 under which the Southeastern Universities Research Association (SURA) operates the Thomas Jefferson National Accelerator Facility.

[1] A.R. Berdoz et al., Phys. Rev. Lett. 87, 272301 (2001).

[2] W.M. Snow et al., Nucl. Inst. and Meth. A 440729 (2000).

[3] B. Desplanques, J.F. Donoghue, and B.R. Holstein, Ann. Phys. (N.Y.) 124, 449 (1980).

[4] R. Hasty et al., Science 290, 2117 (2000).

[5] D.H. Beck and R.D. McKeown, Ann. Rev. Nucl. Part. Sci. 51, 189 (2001).

[6] J. Carlson, M. Paris, and R. Schiavilla, in preparation.

[7] B. Desplanques, Nucl. Phys. A242, 423 (1975); ibidem A335, 147 (1980).

[8] B.H.J. McKellar, Nucl. Phys. A254, 147 (1975).

[9] E.G. Adelberger and W.C. Haxton, Ann. Rev. Nucl. Part. Sci. 35, 501 (1985).

[10] V.V. Flambaum and D.W. Murray, Phys. Rev. C 56, 1641 (1997).

[11] W.C. Haxton and C.E. Wieman, Ann. Rev. Nuc. Part. Sci. 51, 261 (2001).

[12] W.C. Haxton, C.-P. Liu, and M.J. Ramsey-Musolf, Phys. Rev. C 65, 045502 (2002).

[13] D. Bowman, private communication.

[14] J. Carlson, R. Schiavilla, V.R. Brown, and B.F. Gibson, Phys. Rev. C 63, 024003 (2002).

[15] H.J. Pirner and D.O. Riska, Phys. Lett. B44, 151 (1973). 
[16] R.B. Wiringa, V.G.J. Stoks, and R. Schiavilla, Phys. Rev. C 51, 38 (1995).

[17] R. Machleidt, Phys. Rev. C 63, 024001 (2001).

[18] V.G.J. Stoks, R.A.M. Klomp, C.P.F. Terheggen, and J.J. de Swart, Phys. Rev. C 49, 2950 (1994).

[19] J. Carlson and R. Schiavilla, Rev. Mod. Phys. 70, 743 (1998).

[20] A.E. Cox, S.A.R. Wynchank, and C.H. Collie, Nucl. Phys. 74, 497 (1965).

[21] S.F. Mughabghab, M. Divadeenam, and N.E. Holden, Neutron Cross Sections from Neutron Resonance Parameters and Thermal Cross Sections (Academic Press, London, 1981), http://isotopes.lbl.gov/ngdata/sig.htm.

[22] B. Desplanques, Phys. Lett. B512, 305 (2001).

[23] M. Viviani, A. Kievsky, L.E. Marcucci, S. Rosati, and R. Schiavilla, Phys. Rev. C 61, 064001 (2000).

[24] R. Schiavilla, V.R. Pandharipande, and D.O. Riska, Phys. Rev. C 40, 2294 (1989).

[25] K. Tsushima, D.O. Riska, and P.G. Blunden, Nucl. Phys. A559, 543 (1993).

[26] R.B. Wiringa and S.C. Pieper, Phys. Rev. Lett. 89, 182501 (2002).

[27] L. Diaconescu, R. Schiavilla, and U. van Kolck, Phys. Rev. C 63, 044007 (2001).

[28] W.-Y. P. Hwang and E.M. Henley, Ann. Phys. (N.Y.) 129, 47 (1980).

[29] W.-Y. P. Hwang, E.M. Henley, and G.A. Miller, Ann. Phys. (N.Y.) 137, 378 (1981).

[30] W.C. Haxton, E.M. Henley, and M.J. Musolf, Phys. Rev. Lett. 63, 949 (1989).

[31] M.J. Musolf and B.R. Holstein, Phys. Rev. D 43, 2956 (1991).

[32] D.O. Riska, Nucl. Phys. A 678, 79 (2000).

[33] C.M. Maekawa and U. van Kolck, Phys. Lett. B478, 73 (2000).

[34] S.L. Zhu et al., Phys. Rev. D62, 033008 (2000). 
TABLE I: Total $n p$ capture cross-sections (in mb) and $\vec{n} p$ radiative capture asymmetries (in units of $10^{-8}$ ) in various models. Asymmetries are reported for pion-exchange only and full DDH interactions.

\begin{tabular}{|c|c|c|c|c|}
\hline Interaction & $\begin{array}{r}\text { Cross Sec } \\
\text { Impulse Curr }\end{array}$ & $\begin{array}{l}\text { ction } \\
\text { Full Curr }\end{array}$ & $\begin{array}{r}\text { Asym } \\
\text { pion-only }\end{array}$ & $\begin{array}{l}\text { metry } \\
\text { full DDH }\end{array}$ \\
\hline AV18 & 304.6 & 334.2 & -4.98 & -4.85 \\
\hline NIJM-I & 305.4 & 332.5 & -5.11 & -4.95 \\
\hline Bonn-CD & 306.5 & 331.6 & -4.97 & -4.83 \\
\hline
\end{tabular}

TABLE II: Cumulative contributions to the $\vec{n} p$ radiative capture asymmetry (in units of $10^{-8}$ ) at thermal neutron energies for the AV18 interaction and pion-exchange-only DDH interaction. See text for explanation.

\begin{tabular}{|c|c|c|}
\hline & AV18 (PC) Currents & DDH (PV) Currents \\
\hline Impulse & -15.3 & \\
$+\pi$ & -48.3 & 44.2 \\
$+\rho$ & -40.4 & 44.0 \\
$+p$-dependent & -43.8 & 44.0 \\
\hline
\end{tabular}

TABLE III: Cumulative contributions to the $\vec{n} p$ radiative capture asymmetry (in units of $10^{-8}$ ) at thermal neutron energies for the AV6 interaction and pion-exchange-only DDH interaction. Also listed is the asymmetry obtained with the Siegert form of the $E_{1}$ operator. See text for explanation.

\begin{tabular}{|c|c|c|c|}
\hline & AV6 (PC) Currents & DDH (PV) Currents & Total \\
\hline Impulse & -18.5 & & -18.5 \\
$+\pi$ & -66.0 & 50.6 & -15.4 \\
$+\rho$ & -56.7 & 50.4 & -6.32 \\
\hline Siegert $E_{1}$ & & & -6.15 \\
\hline
\end{tabular}




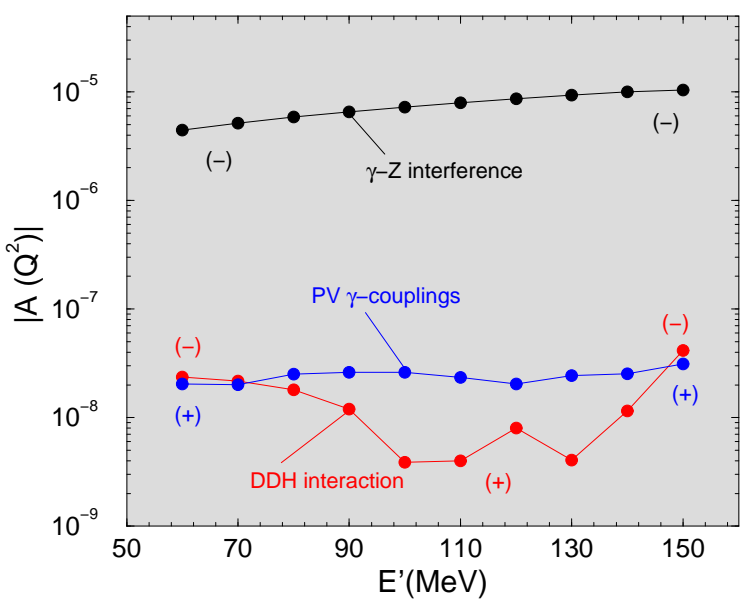

FIG. 1: Contributions (in magnitude) to the longitudinal asymmetry in the electro-disintegration of the deuteron as function of the final electron energy. The kinematical setting corresponds to one of those relevant for the SAMPLE experiments: the initial electron energy is $193 \mathrm{MeV}$ and the electron scattering angle is $145.9^{\circ}$. The $(-)$ and $(+)$ symbols denote the signs of the various contributions. See text for further explanations.

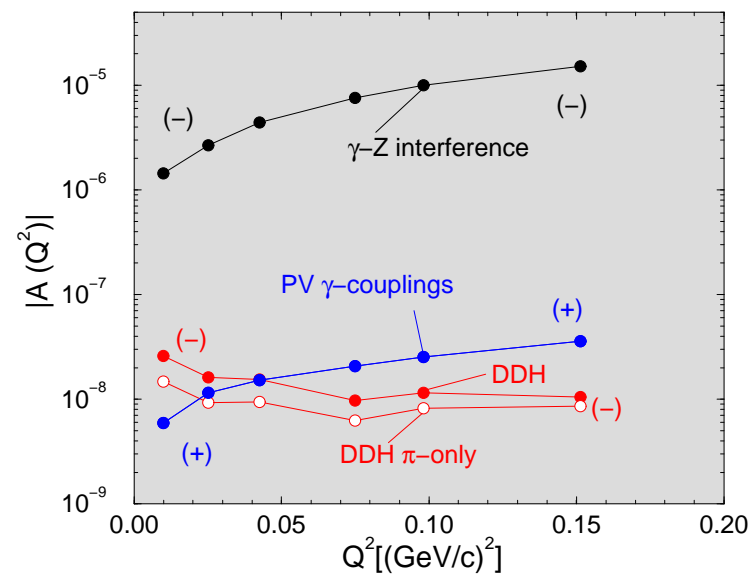

FIG. 2: Contributions (in magnitude) to the longitudinal asymmetry in the deuteron electrodisintegration at the top of the quasi-elastic peak, plotted as function of the four-momentum transfer $Q^{2}$. The $(-)$ and $(+)$ symbols denote the signs of the various contributions. See text for further explanations. 